\title{
Nerve Sheath Tumor of Stomach: Two Cases Report
}

\author{
Hou Dong Zuo ${ }^{a}$, Xiao Ming Zhang ${ }^{\mathrm{a}, \mathrm{b}}$, Zhao Hua Zhai ${ }^{\mathrm{a}}$
}

\begin{abstract}
Nerve sheath tumors of stomach are a group of benign tumors of gastrointestinal tract. Here two cases of this tumor are presented and imaging features are described. A large, irregular well-defined margin mass is demonstrated. Sonography showed heterogeneous hypoechoic lesions. CT features are mainly a large irregular mass with well-defined margin and heterogeneous density, and some calcification can be seen. Contrast-enhanced CT images showed slight inhomogeneous enhancement. On MR images these lesions are demarcated associated with hypointense on T1 weighted imaging and moderate to markedly hyperintense on T2 weighted imaging. Contrast enhanced MRI demonstrated the mass heterogeneous enhancement.
\end{abstract}

Keywords: Nerve sheath tumor; Stomach

\section{Introduction}

Gastrointestinal mesenchymal tumors are a group of tumors, which originate from the mesenchymal stem cells of the gastrointestinal tract, including gastrointestinal stromal tumors (GIST), leiomyomas or leiomyosarcomas or nerve

Manuscript accepted for publication November 24, 2011

${ }^{a}$ Sichuan Key Laboratory of Medical Imaging, Department of Radiology, Affiliated Hospital of North Sichuan Medical College, 63 Wenhua Rd. Nanchong 637000, China

${ }^{\mathrm{b}}$ Corresponding author: Zhang Xiao-ming.

Email: cjr.zhxm@vip.163.com

doi: $10.4021 / \mathrm{jmc} 452 \mathrm{w}$ sheath tumors (i.e. schwannomas). Gastric schwannoma is a very rare spindle cell mesenchymal tumor originating in any nerve that has a Schwann cell sheath, which account for $2 \%$ to $6 \%$ of all stromal tumours of the gastrointestinal tract [1, 2]. It shows distinctive histologic features that separates him from conventional schwannomas. Histologically, gastrointestinal schwannomas are S-100 protein-positive spindle cell tumors with a microtrabecular pattern, peripheral lymphoid cuffing, and occasional germinal centers [3, 4]. Patients with gastrointestinal schwannomas are usually asymptomatic or may present as upper gastrointestinal bleeding or as palpable mass lesions [5-8].

Computed tomography (CT) cross-sectional imaging findings, magnetic resonance imaging (MRI) and ultrasound examination are great useful in the detection and characterization of the tumor and its relation with adjacent organs. We reported two cases with gastrointestinal schwannomas, and presented the features of the tumors on ultrasound, CT and MR imaging.

\section{Case Report}

\section{Case 1}

A 58-year-old female complained repeated dark stools for 3 years and recurrence for 5 days. On physical examination the patient was found a ball-liked large abdominal mass in his left epigastric area without tenderness or rebound tenderness. Laboratory tests were normal. The ultrasound showed a well-defined margin, irregular, heterogeneous mass locating in left epigastric area with the size of approximately 12.3 $\mathrm{cm} \times 8.3 \mathrm{~cm}$, and internal parenchyma was hypoechoic (Fig. 1A). Color Doppler Flow Imaging (CDFI) detected asteria flow (Fig.1B). The patient subsequently performed upper abdominal CT examination in order to evaluate the mass. On $\mathrm{CT}$, the mass was found in the greater curvature of stomach, and showed irregular and well-defined margin with the size of $12.5 \mathrm{~cm} \times 10.5 \mathrm{~cm} \times 8.3 \mathrm{~cm}$. The density of the tumor was heterogeneous associated with some stippled calcification (Fig. 1C, D, E). The tumor showed slight enhancement after contrast enhancement (Fig.1D,E). There was no evidence of 


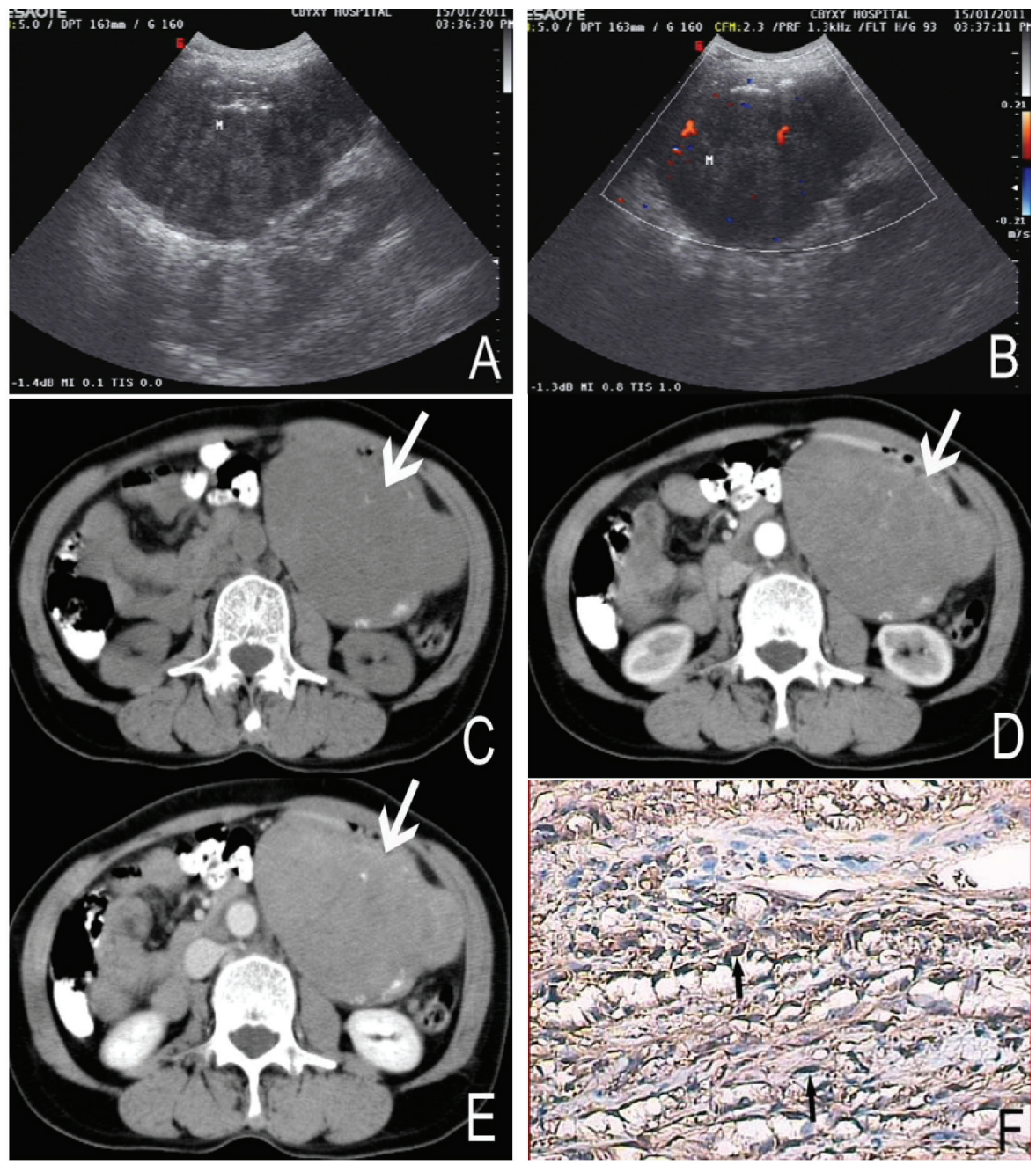

Figure 1. A 58-year-old female with nerve sheath tumor in the greater curvature of stomach. Sonography (A) revealed the mass heterogenous and a low internal echo-level. CDFI (B) detected asteria flow. Non-enhanced CT (C) showed a large, well-defined margin and irregular mass (arrows). The density of the tumor was heterogeneous with some stippled calcification (black arrows). On Contrast-enhanced CT (D, E) the tumor showed slight enhancement. Immunochemistry staining (F) showed the bundles of spindle cell (black arrows) with brown color, positive to S-100 protein (S-100 stain, $\times 200)$.

necrosis within the tumor mass. The inferior aspect of the mass reached the level of body and tail of pancreas. The superior aspect of the mass extended to umbilical level. The body of stomach was squeezed forward by the mass forming arcuated impression.

At exploratory laparatomy, the mass was parenchymatous and found to be adhered to left upper abdominal wall in parietal wall of sinus ventriculi, body and the greater curvature, and there were many hard tubercle on its' surface. Distal stomach resection and Billroth II gastroduodenostomy was performed. The cut surface of this tumor was yellow with a fish flesh appearance and was measured $14 \mathrm{~cm} \times 12$ $\mathrm{cm} \times 9 \mathrm{~cm}$ in size. The histological showed many spindle cells. Immunohistochemical staining revealed strongly positive for S-100 and vimentin, while negative for smooth mus- cle actin, CD117 and CD34 (Fig. 1F).

\section{Case 2}

A 58-year-old female complained repeated epigastric distention and pain accompanied with fever for more than 3 months admitted to our hospital. The other symptoms included loss of appetite, debilitation and diarrhea. Physical examination revealed that left upper quadrant was intumescent, and a moderate hard mass could be touched with tenderness. Laboratory tests were within normal limits. The ultrasound revealed the mass in left upper quadrant with a low echo-level, irregular and well-defined margin. The shape of the mass was like dumb bell (Fig. 2A) and the mass was approximately $20 \mathrm{~cm} \times 12 \mathrm{~cm}$ in size. MRI showed a large, 

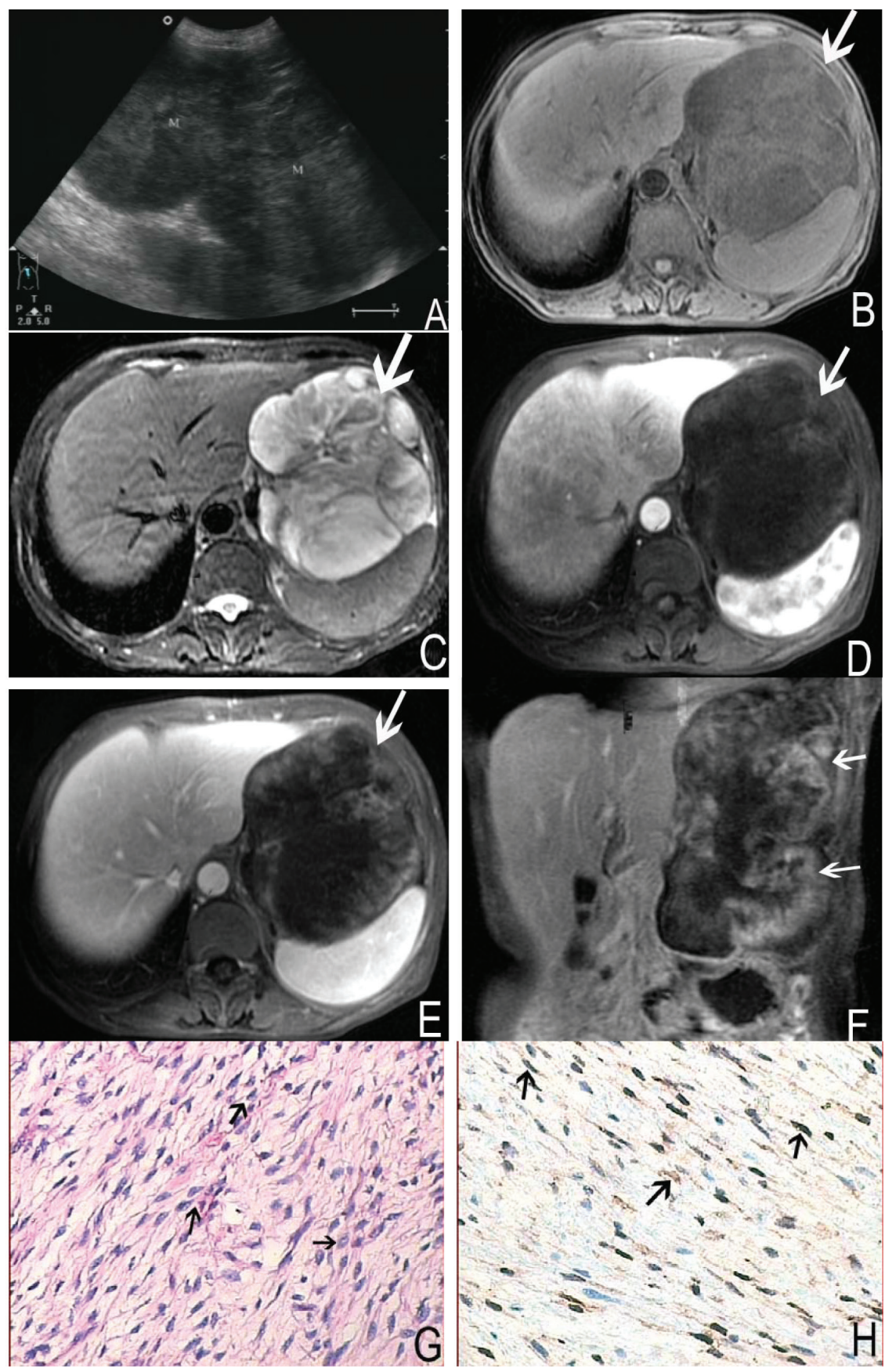

Figure 2. A 58-year-old female with nerve sheath tumor in left upper quadrant. Abdomen sonography (A) revealed a homogeneous, hypoechoic and dumb bell shaped mass. MRI showed a large, irregular and well-defined margin mass (arrows). The mass was hypointense on T1 weighted imaging (B) and moderate to markedly hyperintense on T2 weighted imaging $(C)$. On contrast enhanced MR images, the tumor demonstrated heterogeneous enhancement $(\mathrm{D}, \mathrm{E}, \mathrm{F})$. Histological images $(\mathrm{G})$ and immunochemistry staining $(\mathrm{H})$ showed the bundles of spindle cells (arrows) with brown-tan color, positive to S-100 protein (S-100 stain, $\times 200)$.

irregular and well-defined margin mass in hepatogastric interspace. The superior margin reached diaphragmatic dome, and the inferior margin extended the level of left middle kidney (Fig. 2F). The tumor showed hypointense on T1 weighted imaging and moderate to markedly hyperintense on $\mathrm{T} 2$ weighted imaging. Some septum could be seen and showed more clearly on $\mathrm{T} 2$ weighted images, and the signal intensity appeared to be hypointense, isointense and hyperintense on T2 weighted images (Fig. 2B, C). Contrast enhanced MRI demonstrated heterogeneous enhancement (Fig. 2D, E, F). 
Left diaphragmatic dome was lift for the compression by the mass, left lobe of liver showed invagination. The stomach and pancreas was pushed posteriorly and inferiorly but was clearly separated from the mass.

Operation notes, the mass was adhered to diaphragmatic muscle and spleen, and lobulated in hepatogastric interspace. The amicula was complete. The mass was about $22 \mathrm{~cm} \times 10$ $\mathrm{cm} \times 12 \mathrm{~cm}$ in size and had redundant supplied blood vessels. The cut surface of this mass was yellow-white like fish flesh. The pathological findings showed many spindle cells. Immunohistochemical staining revealed strongly positive for S-100 and vimentin, while negative for smooth muscle actin, CD117 and CD34 (Fig. 2G, H).

\section{Discussion}

Nerve sheath tumors mostly originated in peripheral nerve, very rare in the gastrointestinal tract Schwann cells in the neural plexus, but when they did the most common location was the stomach, and represented $0.2 \%$ of all gastric tumours [9]. Nerve sheath tumor of stomach is also called Gastric schwannomas. Malignant transformation of this is very rare, and only one has been reported in children by Bee et al [10]. It is usually solitary tumor arising from the fundus, body or antrum of the stomach [3-6, 9]. Patients suffered nerve sheath tumors are often asymptomatic or subclinical and can be discovered incidentally at laparotomy or radiographically. The most common presenting symptom is an episode of upper gastrointestinal bleeding and epigastric pain $[8,11-13]$. In our cases, symptoms of this two patients are unrepresentative, one tumor is in the greater curvature of stomach and the other is in fundus and body of stomach, both grows exophytically.

Histologically, these moderately cellular neoplasms appear as interlacing woven nests or bundles of spindle cells. Immunohistochemically, GIS show diffuse strong positivity for S-100 and vimentin, and variable positivity for glial fibrillary acidic protein, while they are typically negative for CD34, CD117, desmin, c-Kit and actin [14-16]. And these two cases findings are consistent with the literatures reported.

Although the definitive diagnosis of gastric nerve sheath tumors is determined by pathological examination, it is considerable helpful to gain information through sonography, $\mathrm{CT}$ and MR imaging.

Yoon et al reported nerve sheath tumors of stomach had a homogenous internal echo on sonography findings [14], but in our cases, the mass were hypoechoic and heterogenous. And Color Doppler Flow Imaging (CDFI) detected asteria flow which indicated the mass had poor blood supply in a way.

CT can demonstrate the extent of invasion and help to determine the appearance of a benign versus malignant le- sion [17]. It also can provide important information to distinguish it from leiomyomas and leiomyosarcomas [18]. On CT images, most tumors had well-defined, round, mural masses with homogeneous attenuation [19]. But in our two cases, case one had a large well-defined margin, irregular and heterogeneous mass with some stippled calcification on non-enhanced CT images. Contrast-enhanced CT scanning showed the tumor slight enhancement. This characteristic is distinct from Levy et al report [19].

MRI can delineate well the relationship between the tumor and adjacent structures, which provided valuable information for surgical planning [20]. Karabulut et al [20] reported the tumor was large, discretely marginated, multilobular, the overall signal pattern was low on T1 weighted images and high on T2 weighted images. Post-gadolinium sequences demonstrated slow but fairly uniform enhancement throughout the mass. In our cases, MR images showed these lesions are demarcated, the signal was hypointense on T1 weighted imaging and moderate to markedly hyperintense on T2 weighted imaging. Some septum could be seen and showed more clearly on T2 weighted images, and the signal intensity appeared hypointense, isointense and hyperintense on T2 weighted images. Contrast enhanced MRI demonstrated heterogeneous enhancement.

The imaging appearance of nerve sheath tumors of stomach has been depicted well in this assay. Some characteristics can be seen such as large, irregular, well-defined margin, hypoechoic, heterogeneous associated with some stippled calcification imaging on CT images, hypointense on T1 weighted imaging and moderate to markedly hyperintense on T2 weighted imaging on MRI. Contrast enhanced scanning is slight or heterogeneous enhancement. And these features are consistent with literature reports except for the characteristic of calcification and enhancement. The diagnosis of this tumor should be paid more attention, especially the mass is exophytic when imaging findings show a welldefined, irregular, solid mass adjacent to the stomach.

\section{References}

1. Inagawa S, Hori M, Shimazaki J, Matsumoto S, Ishii H, Itabashi M, Adachi S, et al. Solitary schwannoma of the colon: report of two cases. Surg Today. 2001;31(9):833838.

2. Matsuki A, Kosugi S, Kanda T, Komukai S, Ohashi M, Umezu H, Mashima Y, et al. Schwannoma of the esophagus: a case exhibiting high 18F-fluorodeoxyglucose uptake in positron emission tomography imaging. Dis Esophagus. 2009;22(4):E6-E10.

3. Sarlomo-Rikala M, Miettinen M. Gastric schwannoma-a clinicopathological analysis of six cases. Histopathology. 1995;27(4):355-360.

4. Daimaru Y, Kido H, Hashimoto H, Enjoji M. Benign 
schwannoma of the gastrointestinal tract: a clinicopathologic and immunohistochemical study. Hum Pathol. 1988;19(3):257-264.

5. Miettinen M, Lasota J. Gastrointestinal stromal tumors-definition, clinical, histological, immunohistochemical, and molecular genetic features and differential diagnosis. Virchows Arch. 2001;438(1):1-12.

6. Rymarczyk G, Hartleb M, Boldys H, Kajor M, Wodolazski A. Neurogenic tumors of the digestive tract: report of two cases. Med Sci Monit. 2000;6(2):383-385.

7. Bosolino A, De la Torre A, Ratto R, Marzano C. Gastric schwannoma. Gastroenterol Hepatol. 2010;33(9):686687.

8. Kim SH. Gastric schwannoma. Korean J Gastroenterol. 2009;53(3):141-143.

9. Melvin WS, Wilkinson MG. Gastric schwannoma. Clinical and pathologic considerations. Am Surg. 1993;59(5):293-296.

10. Bees NR, Ng CS, Dicks-Mireaux C, Kiely EM. Gastric malignant schwannoma in a child. Br J Radiol. 1997;70(837):952-955.

11. Prevot S, Bienvenu L, Vaillant JC, de Saint-Maur PP. Benign schwannoma of the digestive tract: a clinicopathologic and immunohistochemical study of five cases, including a case of esophageal tumor. Am J Surg Pathol. 1999;23(4):431-436.
12. Lin CS, Hsu HS, Tsai CH, Li WY, Huang MH. Gastric schwannoma. J Chin Med Assoc. 2004;67(11):583-586.

13. Chaudry NU, Zafar S, Israr ul H. Benign nerve sheath tumor of stomach. J Coll Physicians Surg Pak. 2007; 17(5):277-279.

14. Yoon HY, Kim CB, Lee YH, Kim HG. Gastric schwannoma. Yonsei Med J. 2008;49(6):1052-1054.

15. Abraham SC. Distinguishing gastrointestinal stromal tumors from their mimics: an update. Adv Anat Pathol. 2007;14(3):178-188.

16. Chetty R, Vajpeyi R, Penwick JL. Psammomatous melanotic schwannoma presenting as colonic polyps. Virchows Arch. 2007;451(3):717-720.

17. Bruneton JN, Drouillard J, Roux P, Ettore F, Lecomte P. Neurogenic tumors of the stomach. Report of 18 cases and review of the literature. Rofo. 1983;139(2):192-198.

18. Vinhais SN, Cabrera RA, Nobre-Leitao C, Cunha TM. Schwannoma of the esophagus: computed tomography and endosonographic findings of a special type of schwannoma. Acta radiol. 2004;45(7):718-720.

19. Levy AD, Quiles AM, Miettinen M, Sobin LH. Gastrointestinal schwannomas: CT features with clinicopathologic correlation. AJR Am J Roentgenol. 2005;184(3):797-802.

20. Karabulut N, Martin DR, Yang M. Case report: gastric schwannoma: MRI findings. $\mathrm{Br} \mathrm{J}$ Radiol. 2002;75(895):624-626. 